This item was submitted to Loughborough's Research Repository by the author.

Items in Figshare are protected by copyright, with all rights reserved, unless otherwise indicated.

\title{
The role of extra-corporeal shockwave therapy (ESWT) plus rehabilitation for patients with chronic greater trochanteric pain syndrome (GTPS): A case series assessing effects on pain, sleep quality, activity and functioning
}

\section{PLEASE CITE THE PUBLISHED VERSION}

http://dx.doi.org/10.1080/17536146.2016.1195623

\section{PUBLISHER}

(c) Taylor and Francis

\section{VERSION}

AM (Accepted Manuscript)

\section{PUBLISHER STATEMENT}

This work is made available according to the conditions of the Creative Commons Attribution-NonCommercialNoDerivatives 4.0 International (CC BY-NC-ND 4.0) licence. Full details of this licence are available at: https://creativecommons.org/licenses/by-nc-nd/4.0/

\section{LICENCE}

CC BY-NC-ND 4.0

\section{REPOSITORY RECORD}

Wheeler, Patrick C., and Chloe Tattersall. 2019. "The Role of Extra-corporeal Shockwave Therapy (ESWT) Plus Rehabilitation for Patients with Chronic Greater Trochanteric Pain Syndrome (GTPS): A Case Series Assessing Effects on Pain, Sleep Quality, Activity and Functioning". figshare. https://hdl.handle.net/2134/26239. 
The role of Extra-Corporeal Shockwave Therapy (ESWT) plus rehabilitation for patients with chronic Greater Trochanteric Pain Syndrome (GTPS), a case series assessing effects on pain, sleep quality, activity and functioning

\section{ABSTRACT:}

2 Background: Greater Trochanteric Pain Syndrome (GTPS) is a common cause of

3 lateral hip pain, most commonly affecting female patients aged 40-60, and which can

4 have a significant impact on patients' quality of life. Extra-corporeal shockwave

5 therapy (ESWT) alongside a structured rehabilitation programme has been shown in

6 previous research studies to have a significant improvement in patient's levels of pain,

7 although it is unclear if this then leads to improved level of global functioning or

8 activity. This case series examines the change in a range of patient reported outcome

9 measures (PROMs) following shockwave therapy as well as the frequency of self-

10 reported side-effects.

12 Methods: Patients undergoing extra-corporeal shockwave therapy for Greater

13 Trochanteric Pain Syndrome were identified from case logs from a single NHS clinic.

14 Patients completed a range of validated patient-rated outcome measures at baseline

15 and at subsequent follow-up appointments. These include measures of pain, and

16 measures of local hip functioning (Oxford Hip Score - OHS, Non-Arthritic Hip Score

17 - NAHS), global functioning (EQ-5D-5L), sleep quality (Pittsburgh Sleep Quality

18 Index - PSQI), anxiety and depressive symptoms (Hospital Anxiety \& Depression

19 Scale - HADS), and activity levels (International Physical Activity Questionnaire -

20 IPAQ.)

22 Results: 45 patients who completed ESWT for greater trochanteric pain syndrome

23 were identified; with a median follow-up duration of 189 days. Side-effect incidence

24 was low, with $<10 \%$ reporting bruising, and no patients withdrew due to side-effects. 
The role of Extra-Corporeal Shockwave Therapy (ESWT) plus rehabilitation for patients with chronic Greater Trochanteric Pain Syndrome (GTPS), a case series assessing effects on pain, sleep quality, activity and functioning

25

"Average" and "worst" self-reported pain values improved significantly from baseline at all time-periods studied; $6.3 / 10$ and $8.2 / 10$ to $3.8 / 10$ and 5.4/10 at three-months respectively, correlating to an improvement of about a third. At three months $63 \%$ of patients were either satisfied or very satisfied, and $70 \%$ would recommend the procedure, these figures increased at six-months. Sleep quality, measures of local hip functioning, and depressive symptoms all improved consistently across different timepoints, however activity levels and global health markers showed less evidence of improvement.

Conclusions: Extra-Corporeal Shockwave Therapy is known to be effective in patients with Greater Trochanteric Pain alongside a structured rehabilitation programme, and this case series is in keeping with the available evidence. This series demonstrates benefits across different areas of functioning. However, in this series physical activity levels did not increase even though pain decreases. As staying active has numerous health benefits further targeted intervention to address this alongside the reduction of pain may be required for optimal health outcomes. 
The role of Extra-Corporeal Shockwave Therapy (ESWT) plus rehabilitation for patients with chronic Greater Trochanteric Pain Syndrome (GTPS), a case series assessing effects on pain, sleep quality, activity and functioning

\section{Background}

44 Greater Trochanteric Pain Syndrome (GTPS) is a common cause of lateral hip pain, with a incidence of 1.8/1000 patients per year in primary care,[1] and accounts for $20 \%$ of referrals to some orthopaedic spinal centres.[2] GTPS is commoner in women than men, and most commonly affects women in their $4^{\text {th }}$ and $5^{\text {th }}$ decade. $[3,4]$ Greater trochanteric pain is known to be commoner in patients with pre-existing low back pain, osteoarthritis of the knee (of either leg), and iliotibial band (ITB) pain, but conflicting evidence exists as to whether it is commoner in overweight or obese patients.[4, 5]

Greater trochanteric pain syndrome (GTPS) has held a range of alternative names over the last few decades, indicating the on-going confusion as to the pathological processes involved. These alternative terminologies have focussed on different anatomical structures (trochanteric bursitis, or gluteus medius tendinosis), or are region based (lateral hip pain, greater trochanteric pain.) Different structures have been postulated to be involved, with attention moving away from the bursae themselves, which had been the original focus, and more towards the tendons of the abductors and external rotators, especially gluteus medius.[4, 6, 7] This varied nomenclature can cause confusion to patients and clinicians alike, and for the purposes of this article the phrase "greater trochanteric pain" will be used, although the criticisms and limitations of this terminology are accepted. 
The role of Extra-Corporeal Shockwave Therapy (ESWT) plus rehabilitation for patients with chronic Greater Trochanteric Pain Syndrome (GTPS), a case series assessing effects on pain, sleep quality, activity and functioning

65 Regardless of the terms used, this condition describes an area of reproducible pain over the area of the greater trochanter, which can spread to the buttock, or upper

67 lateral thigh with occasional spread further down the leg, and which can mimic other conditions including nerve root impingement, spinal problems or hip joint pathologies.[2, 4] Examination typically reveals maximal tenderness in the posterolateral area of the greater trochanter, however the majority of clinical tests have been found to have limited sensitivity for greater trochanteric pain and are poorly able to differentiate this from other causes of lateral hip pain.[8] Identifying those patients with lateral hip pain who do not have particular problems putting on shoes \& socks (which may help to differentiate between GTPS and osteoarthritis of the hip), or those whose lateral hip pain is reproduced by the FABERs test, are thought to be the most reliable clinical questions and assessments to discriminate GTPS from other hip pain sources.[8]

Imaging studies have confirmed tendinopathy of the gluteal muscles to be a common finding in patients with buttock, lateral hip and groin pain, with $88 \%$ patients with trochanteric symptoms having MRI evidence of gluteus tendinopathy compared to $50 \%$ of those with hip pain but without specific trochanteric symptoms; this difference was found to be significant.[6, 9] The absence of any peritrochanteric abnormalities on MRI makes greater trochanteric pain syndrome unlikely, however these changes occur in a high proportion of patients without trochanteric pain.

Caution is therefore required in interpreting imaging results in this patient cohort and may be more useful in ruling out other conditions such as osteoarthritis of the hip, or tears of the gluteal tendons.[9] 


\section{The role of Extra-Corporeal Shockwave Therapy (ESWT) plus rehabilitation for patients with chronic Greater Trochanteric Pain Syndrome (GTPS), a case series assessing effects on pain, sleep quality, activity and functioning}

90 Whilst many cases of greater trochanteric pain will settle with simple conservative

91 management options, a study based in primary care has shown that after one year $36 \%$

92 of patients will still suffer with trochanteric pain, and at 5 years this remains $29 \%$

93 indicating the chronic nature of this condition.[1] Patients who received a

94 corticosteroid injection had a 2.7-fold chance of recovery compared to those who did

95 not.[1] However one randomised controlled trial that sought to examine the benefits

96 of corticosteroid injection over usual care found a significant benefit favouring

97 injections at 3 months, but by 12 months there was no benefit.[10] In addition to being

98 significantly more expensive, there appears to be no added clinical benefit in guided

99 versus unguided corticosteroid injections, with both often being effective in the short

100 and medium term, and 41\%-47\% patients still benefitting at three months.[11] Other

101 conservative treatment options that have been shown to be effective include

102 physiotherapy, non-steroidal anti-inflammatory drugs, and weight loss.[4] Surgery has

103 been tried in recalcitrant cases and there have been a range of different techniques

104 reported with surgery focussing on the bursa, the tendinopathy, or the ITB

105 components of greater trochanteric pain syndrome, which highlights on-going

106 uncertainties as to the underlying pathologies in this condition.[12-14]

108 Extra-Corporeal Shockwave Therapy (ESWT) can also be used to treat patients with

109 trochanteric pain. This is the use of high-energy, inaudible, sound waves generated

110 externally to the body and which are transmitted through the skin, and are often felt as

111 vibrations. Whilst treatment doses vary, and there is case-control study showing

112 benefits of a single-dose of ESWT in patients with GTPS,[15] ESWT is most 
The role of Extra-Corporeal Shockwave Therapy (ESWT) plus rehabilitation for patients with chronic Greater Trochanteric Pain Syndrome (GTPS), a case series assessing effects on pain, sleep quality, activity and functioning

113 typically performed over three sessions at weekly intervals in order to promote a

114 healing response alongside a structured rehabilitation programme. [3] This has a

115 growing evidence base in the treatment of a number of different tendinopathy

116 conditions, of which Greater Trochanteric Pain Syndrome may be one. Currently,

117 there is limited evidence of benefit from the use of Extra-corporeal Shockwave

118 Therapy (ESWT) specifically in patients with trochanteric pain, with the 2011 NICE

119 guidance (IPG 376) highlighting that overall the evidence was inconclusive.[16]

120 There is some case-series evidence which suggests benefit of ESWT [15], and one

121 randomised control trial found that ESWT was better than physiotherapy or

122 corticosteroid injection at four-months, at fifteen-months there were similar results

123 from ESWT and physiotherapy, and that both were more effective than corticosteroid

124 injections alone.[3] A subsequent systematic review looking at evidence in arrange of

125 lower-limb tendon conditions has suggested that ESWT may be useful in managing

126 patients with greater trochanteric pain syndrome as an alternative to other

127 conservative treatments such as corticosteroid injection.[17] Table 1 displays the

128 published studies involving ESWT for Greater Trochanteric Pain Syndrome, which

129 remain limited in number.

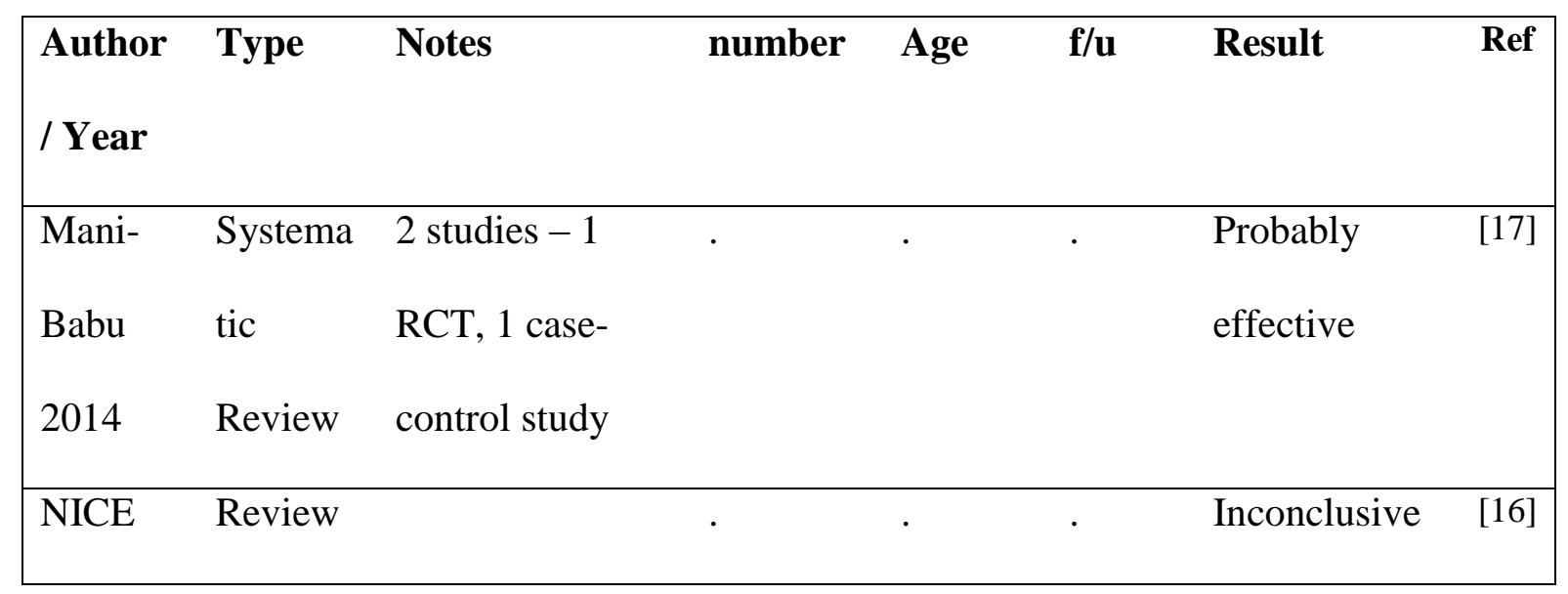


The role of Extra-Corporeal Shockwave Therapy (ESWT) plus rehabilitation for patients with chronic Greater Trochanteric Pain Syndrome (GTPS), a case series assessing effects on pain, sleep quality, activity and functioning

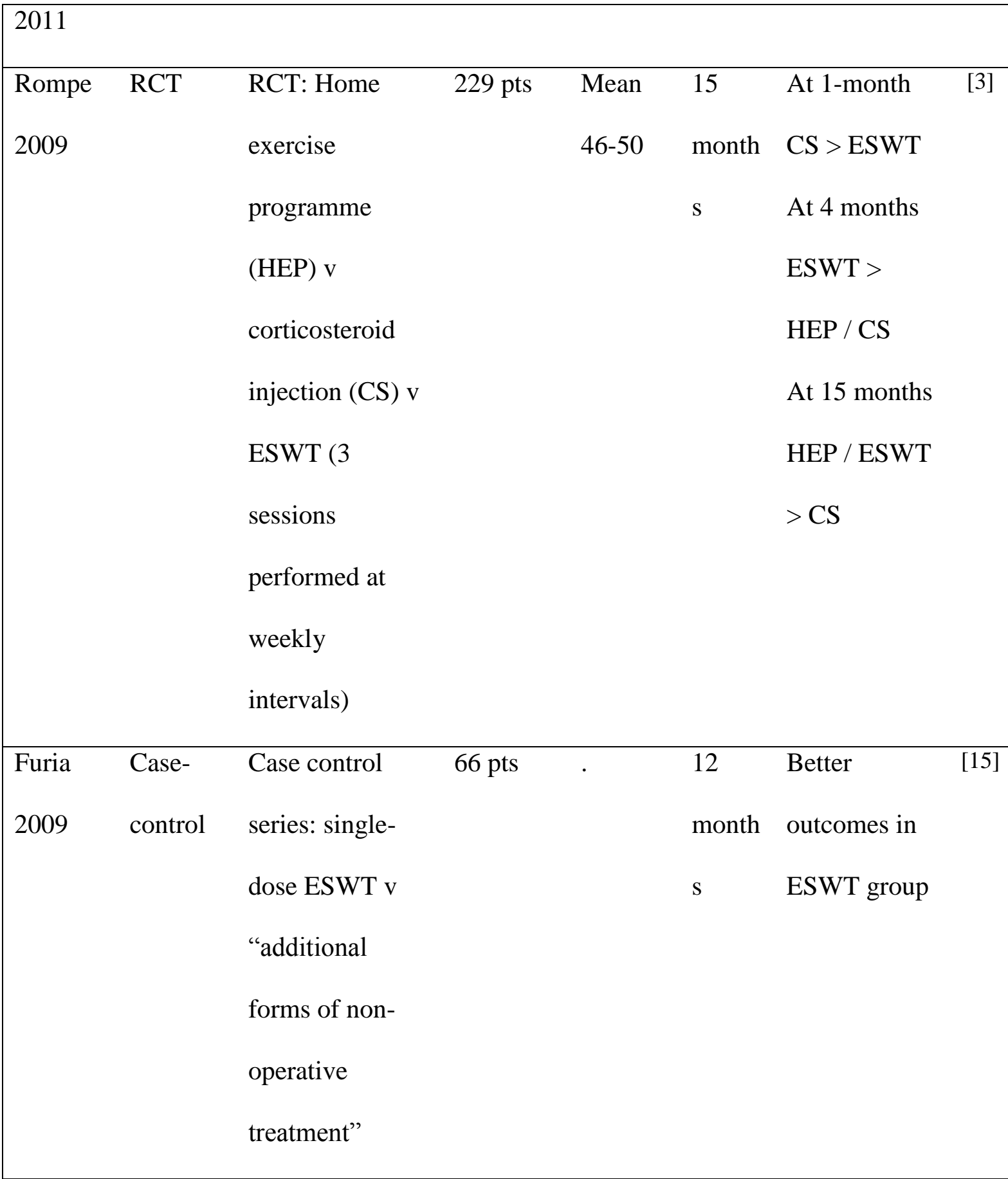

Table 1: Previous published studies investigating effectiveness of ESWT for

\section{GTPS}

133 The side-effect profile from ESWT is favourable, with few serious side-effects

134 reported in most papers across a range of conditions treated.[18] In a placebo- 
The role of Extra-Corporeal Shockwave Therapy (ESWT) plus rehabilitation for patients with chronic Greater Trochanteric Pain Syndrome (GTPS), a case series assessing effects on pain, sleep quality, activity and functioning

135 controlled study of more than 270 patients, reported side-effects included transitory

136 reddening of the skin (21\%) which was harmless and did not lead to treatment

137 cessation, pain (4.8\%), and small haematomas (3\%), in addition there was a

138 possibility of ESWT triggering migraine or possible fainting.[19] The risk of

139 haematoma was reported following the use of a non-MSK specific machine, and

140 newer more MSK-specific ESWT devices, appear to have a safer side-effect

141 profile.[19] Other reports of side-effects from the NICE guidance for lateral hip /

142 trochanteric pain (IPG 376) report than in $2 \%$ of patients there was increased pain of

143 more than 1 day following ESWT treatment, and skin irritation in 33\% of patients at 1

144 month.[16]

145

146 This case series seeks to assess the frequency of side-effects seen and to quantify any

147 changes in pain or other function measures following ESWT in an NHS clinic, and

148 acts as an initial pilot study for further research in this area. 


\section{The role of Extra-Corporeal Shockwave Therapy (ESWT) plus rehabilitation for patients with chronic Greater Trochanteric Pain Syndrome (GTPS), a case series assessing effects on pain, sleep quality, activity and functioning}

151 Methods

152 Patients with chronic Greater Trochanteric Pain Syndrome (GTPS) treated with Extra-

153 Corporeal Shockwave Therapy (ESWT) have been treated by the authors within a

154 single NHS Sports Medicine department in a secondary care hospital in the UK. In

155 line with other hospital procedures, written consent forms are used to record consent

156 before the first session of ESWT. Patients have sessions of ESWT performed by the

157 same practitioner, once per week for three weeks. In keeping with routine use, the

158 energy dose is controlled by the operator to a "maximal comfortably-tolerated"

159 energy dose which was individual for different patients, and varied between sessions.

160 Patients are given standardised post-procedural advice and are advised to avoid

161 NSAIDs for the day of, and a few days after, each session of ESWT.

163 Before undergoing shockwave therapy, patients are taught to perform a structured

164 home exercise programme including flexibility of the lower limb, lumbar mobilisation

165 and range of movement, strengthening of the muscles around the hip including the

166 gluteal muscles associated with GTPS, as well as core stability and proprioception

167 exercises.[3, 20] These exercises are prompted at each of the subsequent clinic visits

168 to promote adherence and facilitate progression. Patients are advised that these

169 exercises can be uncomfortable, particularly to begin with, and are taught how to

170 progress these. To support the use of the home exercise programme, patients are given

171 written sheets discussing these exercises and reminding them of technique and how

172 often these need to be performed for benefit. 
The role of Extra-Corporeal Shockwave Therapy (ESWT) plus rehabilitation for patients with chronic Greater Trochanteric Pain Syndrome (GTPS), a case series assessing effects on pain, sleep quality, activity and functioning

- Data collection

175 Patients complete a structured questionnaire about their symptoms before treatment 176 and at each subsequent follow-up visit. These outcome measures include questions 177 about pain, as well as a range of validated Patient-Rated Outcome Measures (PROMs)

178 which include questionnaires about sleep quality (Pittsburgh Sleep Quality Index 179 PSQI), global function (EQ-5D-5L), specific hip function (Oxford Hip Score - OHS, 180 and the Non-Arthritic Hip Score - NAHS), as well as measures of anxiety and 181 depression symptoms (Hospital Anxiety and Depression Scale - HADS). Lastly 182 questionnaires are used to quantify levels of physical activity. These include the short183 form (7-day recall) version of the International Physical Activity Questionnaire 184 IPAQ, and also two "vital signs" physical activity questions ("On how many days in 185 the last week have you been at least physically active?" and "on how many minutes 186 were you active for?" - multiplying these two figures together to give the number of 187 active minutes in a week.)

189 These measures are all used to examine outcomes following the ESWT procedure.

190 Table 2 displays information for each of the PROMs in use.

\begin{tabular}{llll} 
Outcome measure & Assessing & Scale & Notes \\
\hline Oxford Hip Score & Measure of hip- & Range 0-48 & Higher score \\
(OHS) & specific & indicates better \\
& functioning & self-rated hip \\
& & functioning
\end{tabular}

Non-Arthritic Hip $\quad$ Measure of hip- $\quad$ Total NAHS Higher score 
The role of Extra-Corporeal Shockwave Therapy (ESWT) plus rehabilitation for patients with chronic Greater Trochanteric Pain Syndrome (GTPS), a case series assessing effects on pain, sleep quality, activity and functioning

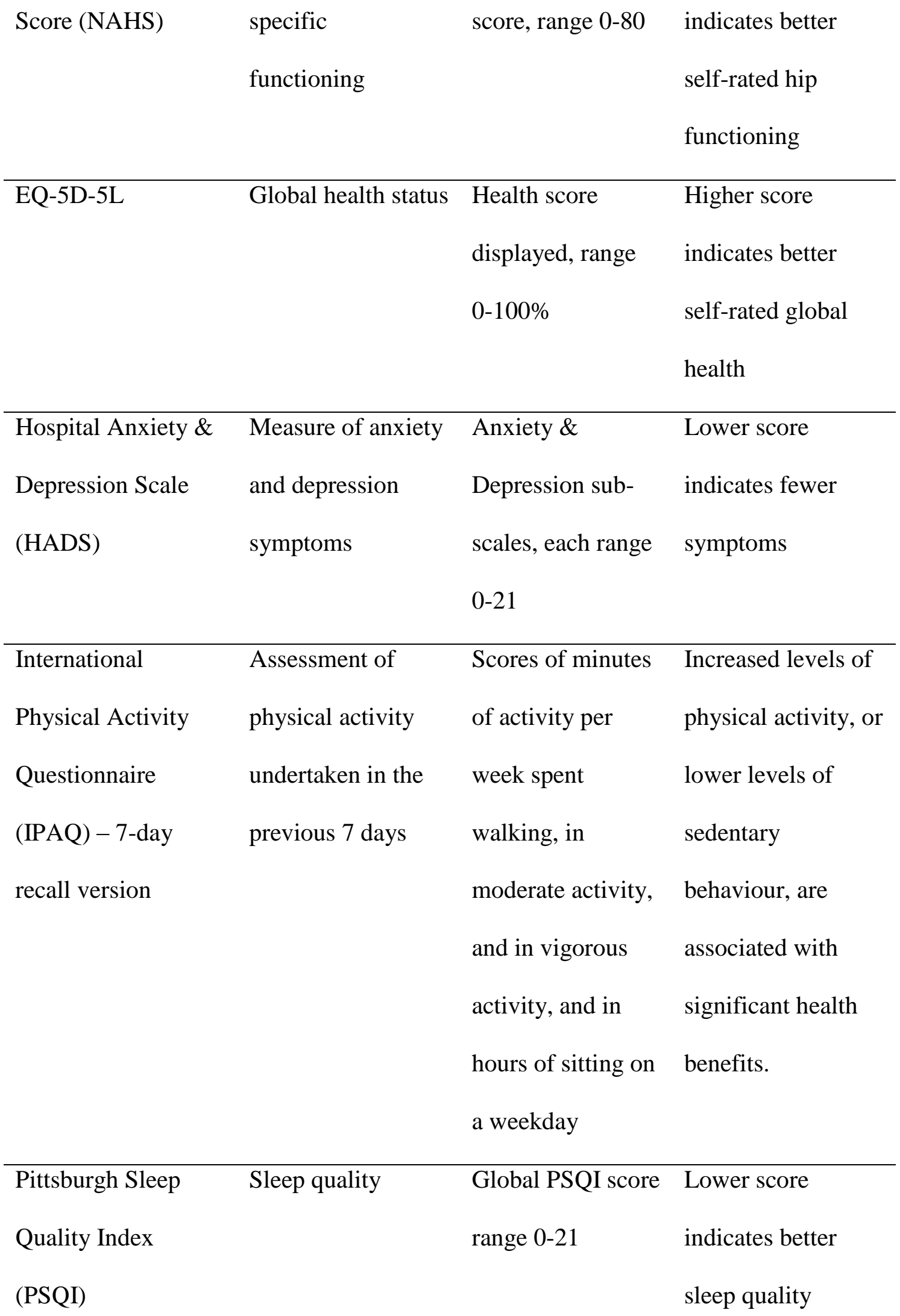

Table 2: Patient-rated Outcome Measures (PROMs) used 
The role of Extra-Corporeal Shockwave Therapy (ESWT) plus rehabilitation for patients with chronic Greater Trochanteric Pain Syndrome (GTPS), a case series assessing effects on pain, sleep quality, activity and functioning

195 Typically, patients are followed up three-months following ESWT, with a proportion 196 also seen at six-weeks where appointment availability allowed. Patients are then 197 routinely followed up after the three-month point if clinically required. Group (NIPAG) and data is recorded here in the format of a service evaluation project and audit; therefore formal NHS ethics permissions were not required.

- Statistical analysis

204 Data was recorded at baseline, and on an on-going basis at clinic follow-up and 205 collated into an Excel spreadsheet (MS Excel from MS Office 2011, version 14.5.7) 206 and analysed in SPSS (IBM SPSS Statistics, version 22). From this dataset the 207 majority of the outcome measures are scale data. Comparisons were made between 208 the baseline data and data from the six-week, the three-month, and where data was 209 present the six-month follow-up appointments. As the sample sizes were small, the 210 Shapiro-Wilk test was used to assess normality and as the majority of the data was

211 found to be not normally distributed the majority of the analysis was performed with 212 non-parametric tests, typically the Wilcoxon Signed-Rank Test to look at pre/post 213 differences. 
The role of Extra-Corporeal Shockwave Therapy (ESWT) plus rehabilitation for patients with chronic Greater Trochanteric Pain Syndrome (GTPS), a case series assessing effects on pain, sleep quality, activity and functioning

\section{Results}

218 A total of 46 patients who underwent Extra-Corporeal Shockwave Therapy (ESWT)

219 for symptoms of trochanteric pain syndrome were identified from procedural logs. All

220 patients were treated with a Chattanooga Intelect RPW rESWT machine using the

221 manufacturer's standard settings for GTPS $(20.0 \mathrm{~Hz}, 2000$ shocks per session, 3

222 treatment sessions at weekly intervals.) The energy intensity was controlled by the

223 performing practitioner based on patient comfort. (The mean(SD) figures were

224 ESWT1 $=2.37(0.27)$ bar, ESWT2 = 2.94(0.41) bar, and ESWT3 = 3.44(0.52) bar)

In addition to the ESWT treatment, all patients were given a structured home exercise

227 programme to complete with written supporting material discussing progressing this

228 as a part of their treatment.

withdrew from ESWT after their second session of treatment as she has been involved

232 in a road traffic collision (unconnected with her trochanteric pain or the ESWT

233 treatment) and was unable to attend the final treatment session due to her other

234 injuries. The data for this patient was therefore removed from analysis.

236 At least one set of follow-up results were available for all of the 45 patients that

237 completed ESWT. Normally patients are invited for follow-up at six-weeks, and

238 three-months following ESWT, and depending on symptoms also some are seen at

239 six-months. Not all patients attended for a six-week appointment post-ESWT due to

240 appointment scheduling and patient availability, with results available for 28/45 
The role of Extra-Corporeal Shockwave Therapy (ESWT) plus rehabilitation for patients with chronic Greater Trochanteric Pain Syndrome (GTPS), a case series assessing effects on pain, sleep quality, activity and functioning

241 patients $(62 \%)$ at the six-week time point, and 44/45 (98\%) at the three-month time-

242 point, which is set as the primary outcome period.

244 Depending on the level on on-going symptoms at three-months, some patients also

245 had a six-month follow-up appointment booked, whereas others were left with an

246 open appointment for them to contact the department if there were problems or

247 questions. In total there were $27 / 45$ responses from patients at six-months (60\%),

248 however these figures may be skewed by the presence of on-going symptoms at three-

249 months and may represent those with either poorer or slower outcomes.

- Patients

25236 of the 45 patients $(80 \%)$ who completed all three ESWT treatments for

253 trochanteric pain were female, and the majority of both male and female patients were

254 either overweight or obese. There was an average(SD) age of 60.9 (15.4) years, with

255 the youngest patient being 20 and the oldest being 86 years old. There was a mean

256 duration of symptoms of 43 months before trying ESWT, however this was skewed

257 by two patients having symptoms for ten years prior to ESWT, with the median

258 duration of symptoms being 30 months.

259

260 Table 3 displays the demographic information for the patients in this series. Figures

261 displayed are mean(SD)

262

$$
\begin{aligned}
& \mathrm{n}=\text { Age Height Weight BMI \%BMI \%BMI Symptom } \\
& \text { (m) } \quad(\mathrm{kg}) \quad\left(\mathrm{kg} / \mathrm{m}^{2}\right) \quad 25-30 \quad 30+\quad \text { duration }
\end{aligned}
$$


The role of Extra-Corporeal Shockwave Therapy (ESWT) plus rehabilitation for patients with chronic Greater Trochanteric Pain Syndrome (GTPS), a case series assessing effects on pain, sleep quality, activity and functioning

(months)

\begin{tabular}{lcccccccc}
\hline Male & 9 & 58.8 & 1.76 & 82.8 & 26.9 & $56 \%$ & $11 \%$ & 43.3 \\
& & $(16.4)$ & $(0.09)$ & $(15.4)$ & $(4.7)$ & & & $(28.9)$ \\
\hline Female & 36 & 60.3 & 1.60 & 75.9 & 29.6 & $31 \%$ & $44 \%$ & 43.2 \\
& & $(15.4)$ & $(0.07)$ & $(12.7)$ & $(4.8)$ & & & $(30.5)$
\end{tabular}

\begin{tabular}{lrrrrrrrr}
\hline All & 45 & 60.0 & 1.64 & 77.4 & 29.0 & $36 \%$ & $38 \%$ & 43.2 \\
& & $(15.4)$ & $(0.10)$ & $(13.4)$ & $(4.8)$ & & & $(29.8)$
\end{tabular}

\section{Table 3: patient demographics}

265 Before ESWT was conducted, all patients had been given a home exercise

266 programme; 91\% had received formal physiotherapy input, the remainder had been

267 given exercises and exercise sheets from other consulting healthcare professionals.

268 There was a wide-range of treatment given prior to ESWT, with an average of 3.0

269 corticosteroid injections given by other healthcare professionals to patients prior to

270 referral for ESWT (range 0-8), with patients reporting an average of 3.6 weeks of

271 benefit from their most recent injection (range 0-20 weeks). One patient had

272 previously undergone surgery for their trochanteric symptoms one year prior to being

273 referred for ESWT. All patients had either received an MRI or an ultrasound scan to

274 examine the condition of the relevant muscles / tendons prior to ESWT as a part of

275 the consideration of treatment process.

- Side-effects from ESWT 
The role of Extra-Corporeal Shockwave Therapy (ESWT) plus rehabilitation for patients with chronic Greater Trochanteric Pain Syndrome (GTPS), a case series assessing effects on pain, sleep quality, activity and functioning

278 The incidence of side-effects following ESWT is previously discussed and this was

279 investigated within this case series. Overall incidence of side-effects in this series is

280 low with $7 \%$ and $9 \%$ of patients reporting bruising at the $2^{\text {nd }} / 3^{\text {rd }}$ ESWT treatments

281 respectively, all of which had settled by the six-week and three-month follow-up

282 periods. No patient withdrew due to side-effects. Table 4 displays the incidence of

283 side-effects from the NICE audit criteria for this case series.

$\begin{array}{ccccc}\text { At 2nd } & \text { At 3rd } & \text { At six- } & \text { At } & \\ \text { Treatment } & \text { Treatment } & \text { weeks } & \text { At six- } \\ & & & \text { months } & \\ & & & & \\ (n=45) & (n=45) & (n=28) & (n=43) & (n=27)\end{array}$

Is there any evidence

of local skin

reddening over the

$0 \% \quad 0 \%$

$0 \% \quad 0 \%$

$0 \%$

$0 \%$

treatment site?

Is there any local

bruising or

haematoma over the

$7 \%$

$9 \%$

$0 \%$

$0 \%$

$0 \%$

treatment site?

Is there any evidence

of other local skin /

$0 \%$

$0 \%$

$0 \%$

$0 \%$

$0 \%$

soft tissue damage?

Is there any local

$0 \%$

$0 \%$

$0 \%$

$0 \%$

$0 \%$ 
The role of Extra-Corporeal Shockwave Therapy (ESWT) plus rehabilitation for patients with chronic Greater Trochanteric Pain Syndrome (GTPS), a case series assessing effects on pain, sleep quality, activity and functioning

numbness of the

treated area?

Is there evidence of

rupture of the

structure being

$0 \%$

$0 \%$

$0 \%$

$0 \%$

$0 \%$

treated?

Does the patient

report any increased

stiffness or worsened

$0 \%$

$2 \%$

$0 \%$

$0 \%$

$0 \%$

mobility following

ESWT?

Table 4: Incidence of side-effects following ESWT treatment

- Pain scores

290 The proportion of patients reporting themselves as pain free (VAS $=0$ ) or virtually

291 pain-free (VAS of 0 or 1) at six-weeks was $7 \%$ and $11 \%$ respectively, at three-months

292 the figures were $9 \%$ and $18 \%$ respectively, and at six-months was $19 \%$ and $33 \%$

293 respectively.

295 Overall there was an average reduction in pain as assessed by a 0-10 Visual Analogue

296 Scale (VAS) from 6.3 at baseline, to 4.1 at six-weeks, 3.8 at three-months, and 3.5 at 
The role of Extra-Corporeal Shockwave Therapy (ESWT) plus rehabilitation for patients with chronic Greater Trochanteric Pain Syndrome (GTPS), a case series assessing effects on pain, sleep quality, activity and functioning

six-months post-ESWT. These changes in pain from baseline were found to be

significant at all time-points at six or more weeks. This improvement correlates to an average reduction of pain of about a third at all follow-up time points recorded.

Table 5 displays the self-reported values for "average" pain, self-reported "worst" pain and "stiffness" at baseline and follow-up appointments - all figures are mean(SD) and use a 0-10 visual analogue scale (VAS), with the significance of any changes seen being calculated from baseline values.

305

\begin{tabular}{|c|c|c|c|c|c|c|}
\hline & At & Before & Before & At 6 & At 3 & At 6 \\
& baseline & $2^{\text {nd }}$ & $\mathbf{3}^{\text {rd }}$ & weeks & months & months \\
\hline "Average & $6.3(1.7)$ & $6.4(1.8)$ & $6.0(2.0)$ & 4.1 & 3.8 & 3.5 \\
Pain" & & & & $(2.6) *$ & $(2.7) *$ & $(2.8) *$ \\
$(0-10)$ & & & & & & \\
\hline "Worst Pain" & $8.2(1.2)$ & $\mathrm{x}$ & $\mathrm{x}$ & 6.3 & 5.4 & 5.0 \\
$(0-10)$ & & & & $(2.5) *$ & $(2.9) *$ & $(3.1) *$ \\
\hline "Average & $5.3(2.8)$ & $\mathrm{x}$ & $\mathrm{x}$ & 3.7 & 3.3 & 2.7 \\
Stiffness" & & & & $(3.1) *$ & $(2.6) *$ & $(3.0) *$ \\
(0-10) & & & & & & \\
\hline
\end{tabular}


The role of Extra-Corporeal Shockwave Therapy (ESWT) plus rehabilitation for patients with chronic Greater Trochanteric Pain Syndrome (GTPS), a case series assessing effects on pain, sleep quality, activity and functioning

310 These values are displayed in the Figure 1

311

Figure 1 - Displaying trend for self-reported "worst pain", "average pain" and

315 The changes in "average pain", "worst pain" and "stiffness" were all significantly

316 improved from baseline at all of the follow-up appointments. Although a trend of

317 continued improvement appears to be shown in this series, for the "average pain" and

318 "average stiffness" the differences between the figures at six-weeks and subsequent

319 follow-ups did not reach statistical significance. For the "worst pain" there was a

320 statistically significant improvement in pain at 6-months compared to 6-weeks, but

321 not 3-months. These suggest that the majority of the benefits seen occur in the first 6-

322 weeks after ESWT, although benefits appear to continue beyond this point.

- Sleep disturbance

326 Sleep disturbance is a commonly reported symptom from patients with trochanteric

327 pain syndrome, with pain sleeping on either the affected or the opposite side

328 commonly reported. Sleep quality was assessed by means of the Pittsburgh Sleep

329 Quality Index (PSQI), a patient self-reported questionnaire, at baseline and

330 subsequent follow-up appointments. This questionnaire rates a number of domains of

331 sleep quality and gives individual subs-scales as well as a global score which is

332 displayed here for simplicity, with a lower score indicating better sleep quality

333 overall. 
The role of Extra-Corporeal Shockwave Therapy (ESWT) plus rehabilitation for patients with chronic Greater Trochanteric Pain Syndrome (GTPS), a case series assessing effects on pain, sleep quality, activity and functioning

335 The following table displays the average (SD) global PSQI results obtained at 336 baseline and at follow-up.

\begin{tabular}{lllll} 
& Baseline & 6-weeks & 3-months & 6-months \\
& & & & \\
\hline PSQI (global) & $10.9(3.7)$ & $9.7(4.2) *$ & $9.1(3.7) *$ & $9.0(4.0)^{*}$
\end{tabular}

The changes of global PSQI score from baseline to six-weeks, baseline to threemonths and baseline to six-months, each of between 1.2 and 1.9 points, were all found to be significant $(\mathrm{p}<0.05)$. However, the changes from six-weeks to either three or six-months, and three-months to six-months, were not found to be significantly

343 different.

- Local and global measures of function

346 A range of other patient-rated outcome measures (PROMS) were used to assess

347 outcome including several hip-region PROMS including the Non-Arthritic Hip Score 348 (NAHS) and the Oxford Hip Score (OHS). In addition, as a marker of overall level of 349 health status the EQ-5D-5L was used, and markers of both anxiety and depression 350 were obtained through the use of the Hospital Anxiety and Depression Scale (HADS).

352 Table 7 displays the mean(SD) scores for the different PROMs in use.

Outcome measure $\quad$ Baseline 6-weeks 3-months 6-months 
The role of Extra-Corporeal Shockwave Therapy (ESWT) plus

rehabilitation for patients with chronic Greater Trochanteric Pain

Syndrome (GTPS), a case series assessing effects on pain, sleep quality, activity and functioning

Non-Arthritic Hip

$39.8(12.7) \quad 45.3(17.2) * 50.3(18.6) * 53.6(19.8) *$

Score (NAHS) - total

Oxford Hip Score

$23.4(9.0) \quad 29.3(10.5) * 31.9(10.7) * 33.4(11.1) *$

(OHS)

$\begin{array}{lllll}\text { EQ-5D-5L - \%health } & 67 \%(15 \%) & 72 \%(13 \%) * & 73 \%(19 \%) & 77 \%(15 \%) *\end{array}$

Hospital Anxiety \&

Depression Scale

(HADS) - Anxiety

$7.4(4.3) \quad 6.7(4.1) \quad 6.0(4.0) \quad 5.1(3.3) *$

sub-scale

Hospital Anxiety \&

Depression Scale

(HADS) - Depression

$5.5(3.0)$

$4.1(2.7) *$

$4.4(3.6) *$

$3.7(2.7) *$

sub-scale

353

354

355

356 Many of the differences in scores from either baseline to six-weeks, baseline to three-

357 months, or baseline to six-months showed a significant change $(\mathrm{p}<0.05)$ as indicated

358 above. The depression sub-scale of the Hospital Anxiety and Depression Scale

359 (HADS) was significantly improved following ESWT all time points, whereas the

360 anxiety sub-scale was only significantly different at the six-month follow-up time

361 point. The overall health\% as recorded by the EQ-5D was only improved significantly

362 at six-weeks and six-months, but not at three-months, however the hip-specific 
The role of Extra-Corporeal Shockwave Therapy (ESWT) plus

rehabilitation for patients with chronic Greater Trochanteric Pain

Syndrome (GTPS), a case series assessing effects on pain, sleep quality, activity and functioning

363 patient-rated measures (Oxford Hip Score and the Non-Arthritis Hip Score) were both

364 significantly improved at all time periods studied.

365

366

- Activity levels

367 Patients with trochanteric pain syndrome often report that pain is a barrier to their

368 physical activity, therefore it may be assumed that if pain is reducing then physical

369 activity may increase. The measure this, the rates of physical activity were recorded

370 by using both the short form (7-day recall) International Physical Activity

371 Questionnaire (IPAQ) and also the "Vital signs" questions discussed previously. The

372 results are as displayed in table 8.

Activity measure $\quad$ Baseline 6-weeks 3-months 6-months

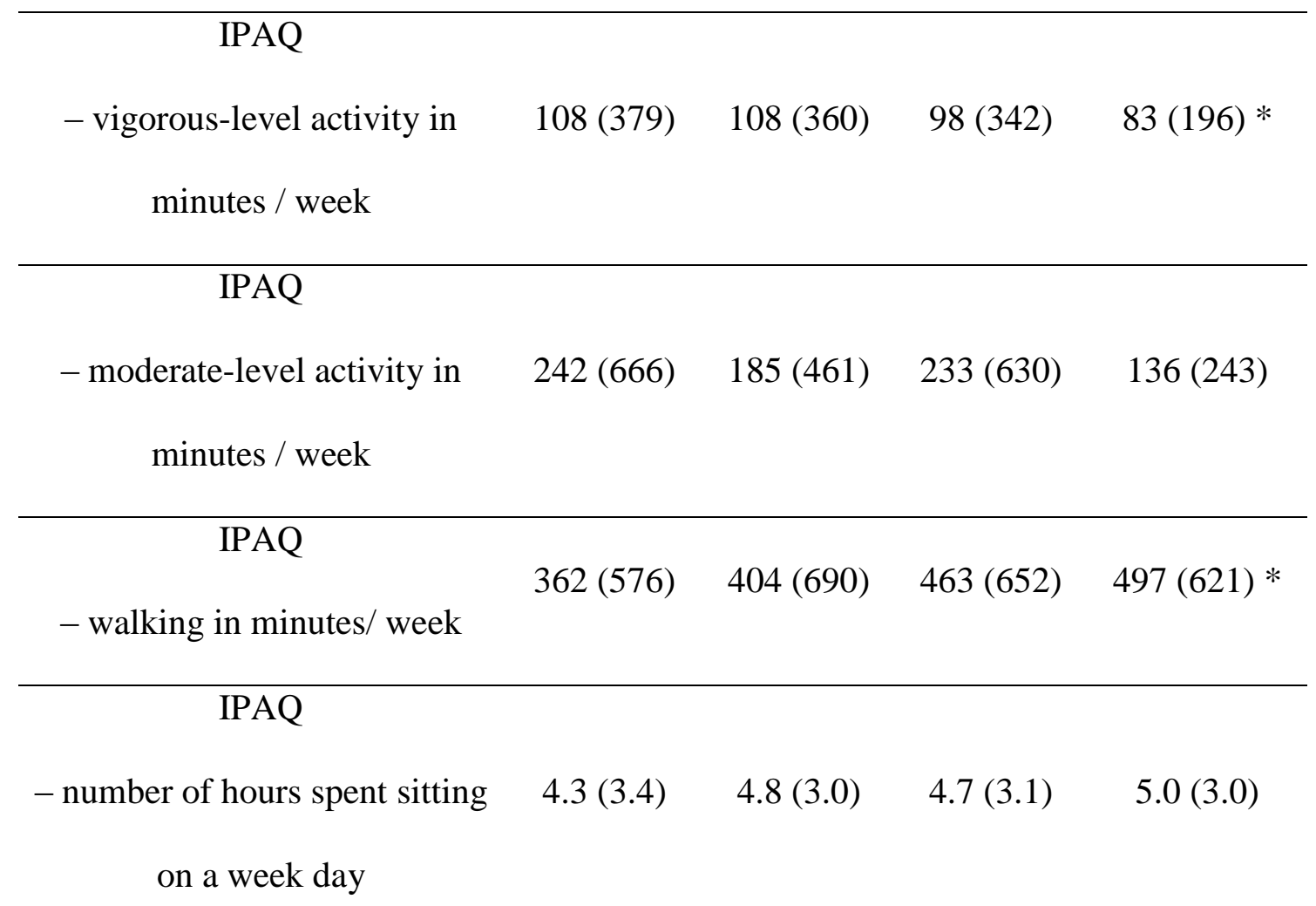


The role of Extra-Corporeal Shockwave Therapy (ESWT) plus rehabilitation for patients with chronic Greater Trochanteric Pain Syndrome (GTPS), a case series assessing effects on pain, sleep quality, activity and functioning

"Vital signs"
- minutes of at least moderate $119(360) \quad 68(124) \quad 95(177) \quad 80(154)$
level of activity / week

Table 8: displaying activity values at baseline and at follow-up

The only measures of physical activity that changed significantly from baseline was

376 decrease in the number of minutes of vigorous activity, and an increase in the number

377 of minutes of walking (both as assessed by the short-form IPAQ) measured at six-

378 months post-ESWT compared to baseline the clinical implications of which are

379 unclear.

- Further intervention rates

382 From the data set available, there was a median follow-up duration of 189 days for

383 this cohort, with a maximum of 315 days. $18 \%$ of patients required consideration of

384 further intervention as a result of persisting symptoms following ESWT, most

385 typically review for surgical intervention or further corticosteroid injection, with $82 \%$

386 of this case series not requiring further intervention during the time period studied.

- Overall satisfaction

389 Overall levels of satisfaction with treatment was assessed on a 5-part Likert scale,

390 with $63 \%$ of patients being either "satisfied" or "very satisfied" at 3-months, and at 6-

391 months this figure had increased to $75 \%$ In addition patients were asked if they would

392 recommend the ESWT treatment to a friend or family member with the same

393 symptoms on a four-part Likert scale. At 3-months $70 \%$ of patients would either 
The role of Extra-Corporeal Shockwave Therapy (ESWT) plus rehabilitation for patients with chronic Greater Trochanteric Pain Syndrome (GTPS), a case series assessing effects on pain, sleep quality, activity and functioning

\begin{tabular}{cccc} 
& $\begin{array}{c}\text { 6-weeks } \\
(\mathbf{n = 1 8})\end{array}$ & $\begin{array}{c}\text { 3-months } \\
(\mathbf{n = 4 0})\end{array}$ & $\begin{array}{c}\text { 6-months } \\
(\mathbf{n = 2 4})\end{array}$ \\
\hline Very satisfied & $39 \%$ & $45 \%$ & $50 \%$ \\
\hline Satisfied & $39 \%$ & $18 \%$ & $25 \%$ \\
\hline Neutral & $11 \%$ & $28 \%$ & $4 \%$ \\
\hline Dissatisfied & $6 \%$ & $5 \%$ & $4 \%$ \\
\hline Very dissatisfied & $6 \%$ & $5 \%$ & $47 \%$ \\
\hline
\end{tabular}

Table 9: "On the basis of your results currently, how satisfied are you

$$
\text { with the results that you have had so far?" }
$$

6-weeks

$(\mathbf{n}=\mathbf{2 0})$ 3-months

$(n=43)$ 6-months

$(\mathbf{n}=\mathbf{2 5})$

\begin{tabular}{cccc}
\hline Yes, definitely & $55 \%$ & $49 \%$ & $64 \%$ \\
\hline Yes, probably & $20 \%$ & $21 \%$ & $16 \%$ \\
\hline Maybe & $20 \%$ & $23 \%$ & $16 \%$ \\
\hline No & $5 \%$ & $7 \%$ & $4 \%$
\end{tabular}

Table 10: "On the basis of your results so far, would you recommend 


\section{The role of Extra-Corporeal Shockwave Therapy (ESWT) plus rehabilitation for patients with chronic Greater Trochanteric Pain Syndrome (GTPS), a case series assessing effects on pain, sleep quality, activity and functioning}

405

406

\section{Discussion}

This case series demonstrates that the majority of patients report improvement in their symptoms. At three-months nearly two-thirds of patients are satisfied with the results of their treatment and $70 \%$ would recommend the treatment to a friend or family member with the same symptoms. The data from this case series, and the previous published work on this topic suggest that Extra-Corporeal Shockwave Therapy can be an effective treatment for a number of patients with recalcitrant Greater Trochanteric Pain Syndrome, which has not settled with other simple conservative measures, and one which is worth considering in care pathways, access permitting. Patients in this case series had a range of symptom duration and different treatments tried prior to referral for ESWT. There was no difference found in reported success or improvement in pain levels for those with symptoms of 18 months or less, compared to those with symptoms greater than 18 months suggestive that ESWT is worth considering in appropriate patients even with long-standing symptoms.

Causality of benefit cannot be proven from this case series design of study alone, but these findings of improvement are in keeping with other published literature. The figures at three-months are the primary end-point with the highest proportion of respondents and patients are typically seen at six-months only if they have on-going or slow resolving symptoms or other concerns. It is possible therefore that even with the figures appearing to have improved at six-months from the three-month period, although this did not necessarily reach statistical significance, these may underestimate health benefits due to selection bias, with patients that are doing well 


\section{The role of Extra-Corporeal Shockwave Therapy (ESWT) plus rehabilitation for patients with chronic Greater Trochanteric Pain Syndrome (GTPS), a case series assessing effects on pain, sleep quality, activity and functioning}

not returning at six-months. The magnitude of benefits may be greater than seen here, and this is worth consideration in further research.

The side-effect profile reported in this case series shows that the incidence of sideeffects from ESWT treatment for Greater Trochanteric Pain Syndrome is relatively low, with no patients in this series failing to complete treatment due to side-effects, and less than $10 \%$ reported bruising. This is a lower figure that quoted in other sources, and this may represent the use of a modern and specific ESWT machine.

This case series has demonstrated an overall average improvement of at least a third in symptoms of pain and stiffness as reported by the patients, as well as improvements in a wide range of other measures of function. The use of simple pain-scores is a very crude outcome measure, and this series has used a range of validated patient-rated outcome measures (PROMs) including specific measures of hip function, and also global measures of function. This holistic view of patient function goes far beyond the use of simple pain-rating tools and should be considered in further work to identify the most relevant outcome measures. Some measures of both local and general functioning have significantly improved, although not all reported benefits. Mood disturbance with both anxiety and depression features as assed by the Hospital Anxiety and Depression Scale (HADS) showed significant improvements at a number of time-periods. Sleep disturbance is an often-reported symptom of Greater Trochanteric Pain Syndrome, and this case series has demonstrated an improvement in sleep quality, as assessed by the PSQI questionnaire at all time-points following treatment. It is accepted that there may be confounding that exists between the various 


\section{The role of Extra-Corporeal Shockwave Therapy (ESWT) plus rehabilitation for patients with chronic Greater Trochanteric Pain Syndrome (GTPS), a case series assessing effects on pain, sleep quality, activity and functioning}

452

453

471 further changes did not reach statistical significance. This is suggestive that the most

472 benefits are gained in the early period following treatment, and it is not clear from this

473 case series when these benefits plateau, meaning that longer-term follow-up may be

474 helpful in identifying final outcome points. A larger series may be able to investigate 
The role of Extra-Corporeal Shockwave Therapy (ESWT) plus rehabilitation for patients with chronic Greater Trochanteric Pain Syndrome (GTPS), a case series assessing effects on pain, sleep quality, activity and functioning

475 this aspect in more detail, as this series was likely to be affected by a $60 \%$ review rate 476 at six-months.

478 In summary this case series has demonstrated a significant improvement in both pain 479 and a wide-range of different outcome measures in the period following extra480 corporeal shockwave therapy and a structured rehabilitation programme. These 481 include a wide range of measures of patent functioning indicating improvement in a 482 range of the symptoms that commonly affect patients with Greater Trochanteric Pain 483 Syndrome. Further work looking at specific benefits of the shockwave itself 484 compared to rehabilitation alone would be useful to quantify this aspect of therapy, 485 and potentially longer-term follow-up may be helpful to see where benefits plateau, 486 which may avoid further interventions being done at a too early time-point, and allow 487 better quality information to be given to patients about longer-term outcomes.

\section{- Abbreviations used}

ESWT - Extra-Corporeal Shockwave Therapy

GTPS - Greater Trochanteric Pain Syndrome

\section{- Ethics approvals}

496 This series is registered at the host NHS Trust as clinical audit. Formal NHS ethics 497 approvals are not required as this constitutes usual treatment. No patient identifiable 498 information is included. 
The role of Extra-Corporeal Shockwave Therapy (ESWT) plus rehabilitation for patients with chronic Greater Trochanteric Pain Syndrome (GTPS), a case series assessing effects on pain, sleep quality, activity and functioning

503 Not applicable - No patient identifiable information is included.

The raw data contained in this publication is not being made publically available at host trust audit team as per local policy.

513 The authors have no potential conflicts of interest to declare

516 No funding sources to declare. Clinical audit performed within employed role at host 517 hospital trust.

\section{- Author contributions}

520 Both authors were involved in the clinical aspects of the cases in this manuscript. The

521 corresponding author took the lead in data analysis and evaluation. The manuscript was prepared and checked by both authors. 
The role of Extra-Corporeal Shockwave Therapy (ESWT) plus rehabilitation for patients with chronic Greater Trochanteric Pain Syndrome (GTPS), a case series assessing effects on pain, sleep quality, activity and functioning

\section{- Acknowledgements}

525 No further acknowledgements are made

\section{REFERENCES}

1. Lievense, A., et al., Prognosis of trochanteric pain in primary care. British Journal of General Practice, 2005. 55(512): p. 199-204.

533

2. Tortolani, P.J., J.J. Carbone, and L.G. Quartararo, Greater trochanteric pain syndrome in patients referred to orthopedic spine specialists. The Spine Journal, 2002. 2(4): p. 251-254.

3. Rompe, J.D., et al., Home Training, Local Corticosteroid Injection, or Radial Shock Wave Therapy for Greater Trochanter Pain Syndrome. The American Journal of Sports Medicine, 2009. 37(10): p. 1981-1990.

4. Williams, B.S. and S.P. Cohen, Greater Trochanteric Pain Syndrome: A Review of Anatomy, Diagnosis and Treatment. Anesthesia \& Analgesia, 2009. 108(5): p. 1662-1670.

5. Segal, N.A., et al., Greater Trochanteric Pain Syndrome: Epidemiology and Associated Factors. Archives of Physical Medicine and Rehabilitation, 2007. 88(8): p. 988-992.

6. Kingzett-Taylor, A., et al., Tendinosis and tears of gluteus medius and minimus muscles as a cause of hip pain: MR imaging findings. American Journal of Roentgenology, 1999. 173(4): p. 1123-1126.

7. Silva, F., et al., Trochanteric Bursitis: Refuting the Myth of Inflammation.

JCR: Journal of Clinical Rheumatology, 2008. 14(2): p. 82-86

8. Fearon, A.M., et al., Greater trochanteric pain syndrome: defining the clinical syndrome. British Journal of Sports Medicine, 2013. 47(10): p. 649-653.

9. Blankenbaker, D.G., et al., Correlation of MRI findings with clinical findings of trochanteric pain syndrome. Skeletal Radiology, 2007. 37: p. 903-909.

10. Brinks, A., et al., Corticosteroid Injections for Greater Trochanteric Pain Syndrome: A Randomized Controlled Trial in Primary Care. The Annals of Family Medicine, 2011. 9(3): p. 226-234.

11. Cohen, S.P., et al., Comparison of fluoroscopically guided and blind corticosteroid injections for greater trochanteric pain syndrome: multicentre randomised controlled trial. British Medical Journal, 2009. 338: p. b1088. 
The role of Extra-Corporeal Shockwave Therapy (ESWT) plus rehabilitation for patients with chronic Greater Trochanteric Pain Syndrome (GTPS), a case series assessing effects on pain, sleep quality, activity and functioning

561

562

563

564

565

566

567

568

569

570

571

572

573

574

575

576

577

578

579

580

581

582

583

584

585

586

587
12. Fox, J.L., The role of arthroscopic bursectomy in the treatment of trochanteric bursitis. Arthroscopy: The Journal of Arthroscopic \& Related Surgery, 2002. 18(7): p. 1-4.

13. Craig, R.A., et al., Iliotibial band Z-lengthening for refractory trochanteric bursitis (greater trochanteric pain syndrome). ANZ Journal of Surgery, 2007. 77(11): p. 996-998.

14. Voos, J.E., et al., Arthroscopic Anatomy and Surgical Techniques for Peritrochanteric Space Disorders in the Hip. Arthroscopy: The Journal of Arthroscopic \& Related Surgery, 2007. 23(11): p. 1246.e1-1246.e5.

15. Furia, J.P., J.D. Rompe, and N. Maffulli, Low-Energy Extracorporeal Shock Wave Therapy as a Treatment for Greater Trochanteric Pain Syndrome. The American Journal of Sports Medicine, 2009. 37(9): p. 1806-1813.

16. NICE, Extracorporeal shockwave therapy for refractory greater trochanteric pain syndrome. (Interventional procedure guidance 376). 2011, National Institute for Health and Clinical Excellence.

17. Mani-Babu, S., et al., The Effectiveness of Extracorporeal Shock Wave Therapy in Lower Limb Tendinopathy: A Systematic Review. The American Journal of Sports Medicine, 2015. 43(3): p. 752-761.

18. Wang, C.J., Extracorporeal shockwave therapy in musculoskeletal disorders. J Orthop Surg Res, 2012. 7: p. 11.

19. Haake, M., et al., Side-effects of extracorporeal shock wave therapy (ESWT) in the treatment of tennis elbow. Archives of Orthopaedic and Trauma Surgery, 2002. 122(4): p. 222-228.

20. Mulligan, E.P., E.F. Middleton, and M. Brunette, Evaluation and management of greater trochanter pain syndrome. Physical Therapy in Sport, 2015. 16: p. 205-214. 


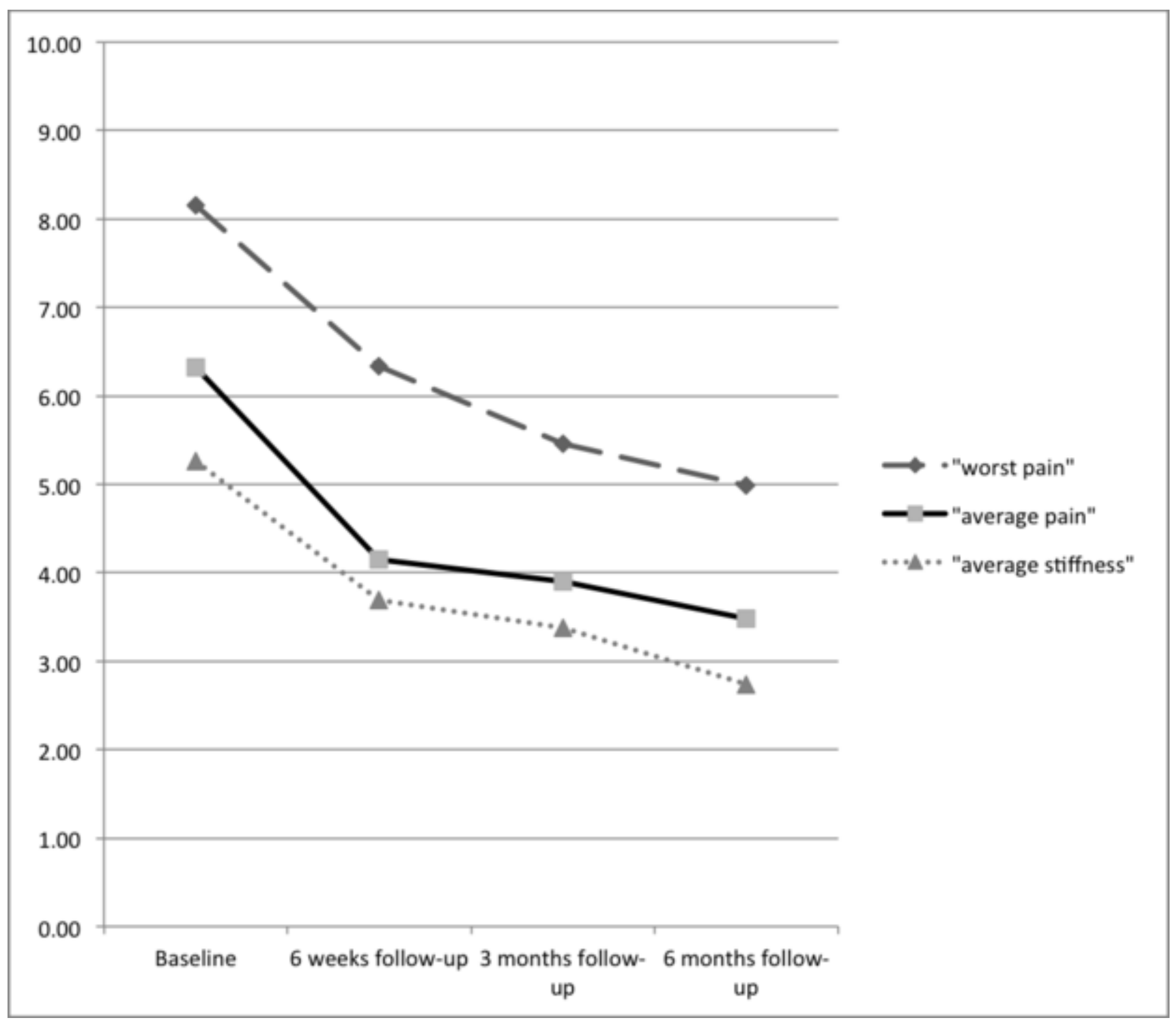

Fisplaying change in pain scores 